EPJ Web of Conferences 24, 07008 (2012)

DOI: 10.1051/epjconf/20122407008

(C) Owned by the authors, published by EDP Sciences - SIF, 2012

\title{
A simple device for the measurement of kerma based on commercial PIN photo diodes
}

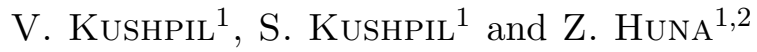 \\ ${ }^{1}$ NPI of ASCR - Rez, Prague, Czech Republic \\ ${ }^{2}$ FNSPE of CTU - Prague, Czech Republic
}

\begin{abstract}
The development of a dose reader for the measurement of the radiation dose from neutron and ionizing radiation is presented. The dose reader (kerma meter) is using as active element commercial PIN diodes with long base, which can provide a maximal dependence of the lifetime of minority carriers from the absorbed dose. We consider a simple theory of PIN diodes for the role of the kerma meter and discuss the idea for optimization of the calibration procedure. The characteristics of the dosimeter were measured for a few types of commercial diodes. The device can be useful for many practical tasks of control for radiation environments in the environment, nuclear physics and for industrial application.
\end{abstract}

\section{Introduction}

PIN diodes are used since 1959 [1] as device for the measurement of doses from fast neutrons and charged particles. In order to measure precisely the absorbed dose few important parameters must be controlled. The control parameters are temperature, the time interval after irradiation of the diode and also the parameters of the test signal (the amplitude of the current and the pulse width). We will consider the optimization of the calibration procedure and the readout of the PIN diode from the point of view of stability

This is an Open Access article distributed under the terms of the Creative Commons Attribution License 2.0, which permits unrestricted use, distribution, and reproduction in any medium, provided the original work is properly cited. 
of the diode bulk temperature. A thermo-stabilization of the P-I-N structure means that the temperature does not exceed a given limit during the complete operation time until the maximum dose is reached. The parameter measurements can be automated and it is possible to do all the important corrections to the results in units of dose. In this article we describe a dosimeter, which by measuring the forward voltage on the PIN diode for a given constant current with changing time pulse width during irradiation is keeping the same value of dissipation of energy.

\section{Short theory for optimal calibration/readout of PIN diodes}

The principle of the use of a PIN diode for the measurement of absorbed dose is described in works $[2,3]$. The total forward voltage can be presented as in equation (1), where $V_{\mathrm{fw}}$ is the total forward voltage, $V_{\mathrm{pn}}$ the junction voltage, and $V_{\mathrm{rb}}$ the voltage on the resistivity of the i-type silicon:

$$
V_{\mathrm{fw}}=V_{\mathrm{pn}}+V_{\mathrm{rb}} .
$$

According to [4] we can present the dependence of each component on total voltage using the semiconductor parameters as reported in equation (2):

$$
\begin{aligned}
V_{\mathrm{fw}}=V_{\mathrm{pn}}+V_{\mathrm{rb}}= & \varphi_{T} \cdot \ln \left[\frac{I(t) \cdot N_{d}(\Phi)}{e \cdot S \cdot N_{i}^{2} \cdot \sqrt{D_{n} \cdot \tau_{n}}}\right] \\
& +\varphi_{T} \cdot \ln \left[\frac{I(t)+B\left(N_{t}[\Phi]\right)}{A\left(N_{d}[\Phi]\right)+B\left(N_{t}[\Phi]\right)}\right] .
\end{aligned}
$$

The dependence of the minority carrier life-time and of the resistivity of silicon on the neutron dose can be obtained from [4]. $N_{d}$ is the concentration of donors in the i-Si, $D_{n}$, and $\tau_{n}$ are the diffusion length and the life-time of the majority carriers in the i-Si, $I(t)$ the forward current at time $t, S$ the area of the P-N junction, $N_{i}$ the intrinsic carrier concentration for silicon, $\phi_{T}=k * T / e, e$ the charge of electron, $k$ the Boltzmann constant, $T$ the temperature of PIN diode, $K_{n d}$ and $K_{n t}$ are the coefficients that describe the dependence of the concentration of the majority carriers and of the traps with energy $0.6 \mathrm{eV}$ on the absorbed dose $\left(N_{d 0}, N_{t 0}\right.$ are the concentrations before irradiation). In the end, for the functions $A$ and $B$ we have

$$
A\left(N_{d}[\Phi]\right)=e \cdot \sqrt{\frac{D_{n}}{\tau(\Phi)}} \cdot \frac{N_{i}^{2}}{N_{d 0} \cdot \exp \left(-K_{n d} \cdot \Phi\right)}
$$




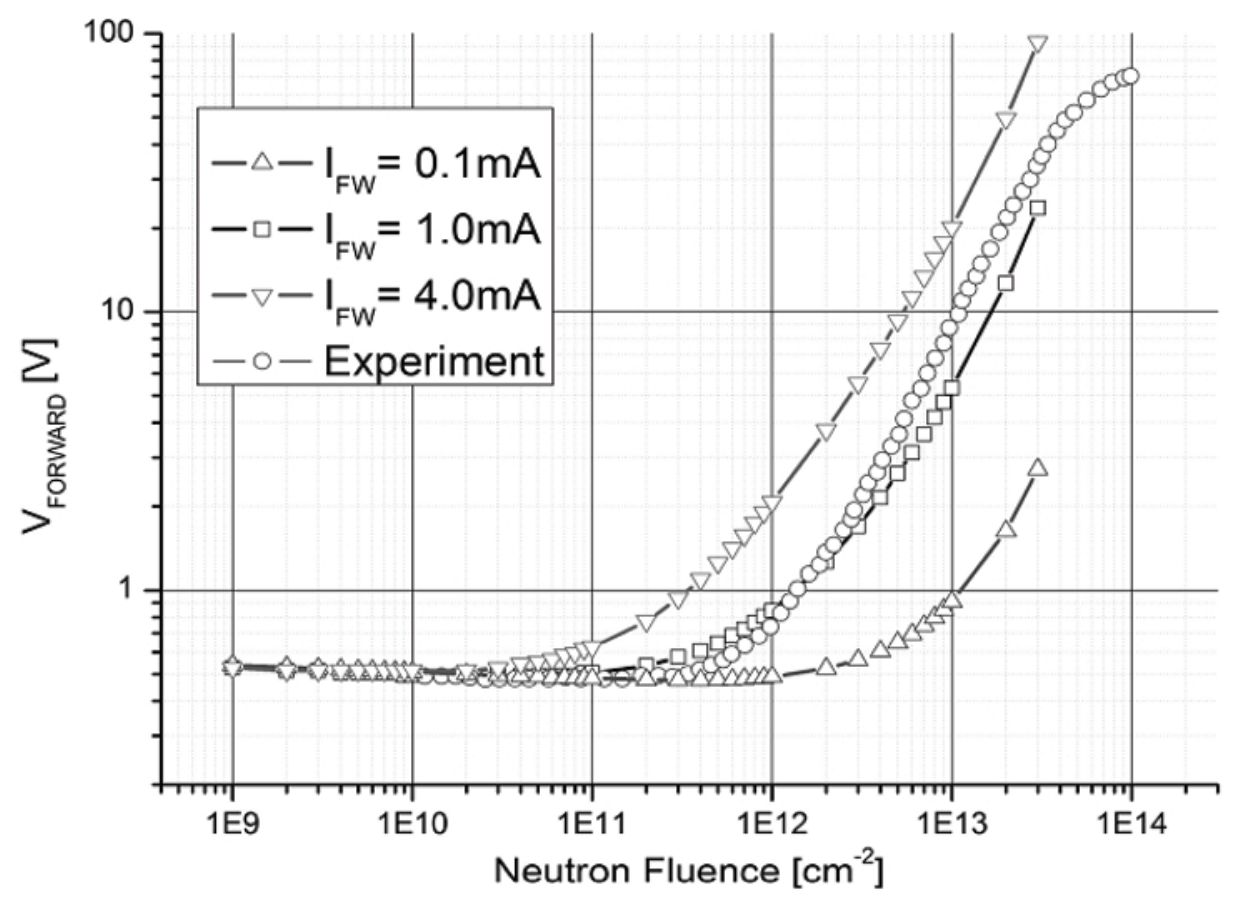

Figure 1: Simulation of the forward voltage as function of the neutron fluence for a BPW34 PIN photo-diode in comparison with the experimental data presented in $[6]$.

and

$$
B\left(N_{t}[\Phi]\right)=e \cdot \frac{L}{2} \cdot N_{i} \cdot N_{t 0} \cdot \exp \left(K_{n t} \cdot \Phi\right) .
$$

We used the data of a commercial PIN photo-diode (BPW34) [6] to estimate the influence of dose on each component of equation (1). The results of the calculation are presented in Figure 1. According to [5] we can estimate the temperature of the passive part of the diode for a given pulse width and constant current. To simplify, we assume that the process of cooling of the diode does not play an important role, and that the temperature of the complete diode is the same as the temperature of the passive part of the diode (the i-type silicon). The differential equation describing the process of heating of the diode in this case can be presented as in equation (3),

$$
\frac{\mathrm{d} T}{\mathrm{~d} t}=\frac{\rho_{n}(T)}{C \cdot p \cdot S^{2}} \cdot I^{2}(t)
$$

where $\rho_{n}(T)$ is the resistivity of silicon, $C$ the specific heat of silicon, $p$ 


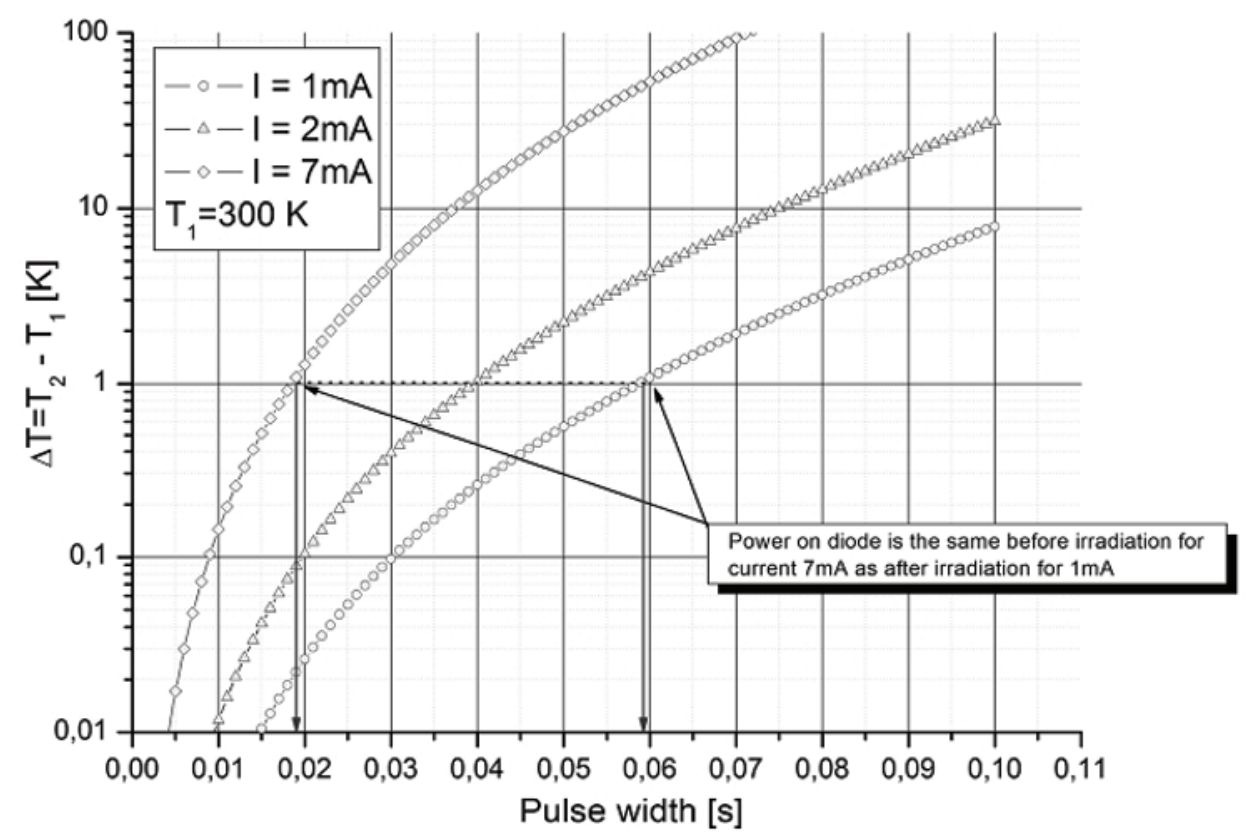

Figure 2: The increase of temperature of a BPW34 PIN photo-diode as function of the pulse width for different forward currents.

the density of silicon, $I(t)$ the forward current of the diode as function of time $t, L$ the length of the passive part of the diode, and $S$ the area of the diode. An accurate measurement of $V_{\mathrm{fw}}$ is possible, if the energy dissipation before irradiation and after irradiation is the same (i.e., the diode bulk is at the same temperature). If the serial resistance of the i-type region before irradiation is $R_{\mathrm{bo}}$ and its resistivity after irradiation is $R_{\mathrm{bi}}$ and $R_{\mathrm{bi}} / R_{\mathrm{bo}}=K$, from the equilibrium of power dissipation we can obtain a relation for the currents $I_{i}=I_{0} / K^{1 / 2}$. From experimental data we have got an upper limit for $K$ (for the PIN diode BPW34 $K$ is less than 60 ). In case $K$ equals 49 the energy dissipation for a current of $1 \mathrm{~mA}$ for $60 \mathrm{~ms}$ will be the same as for a current of $7 \mathrm{~mA}$ for a pulse length of $20 \mathrm{~ms}$.

Results of the simulation are shown in Figures 1 and 2. The simulation of the dependence of the forward voltage versus dose (Figure 1) was obtained under the assumption that during irradiation in silicon only a single trap level is created with energy half of the energy gap of silicon. This could explain the large uncertainties in the calculation in comparison with experimental data. The dependencies presented in Figure 2 can be used for the estimation of the length of the current pulse after irradiation for diodes 


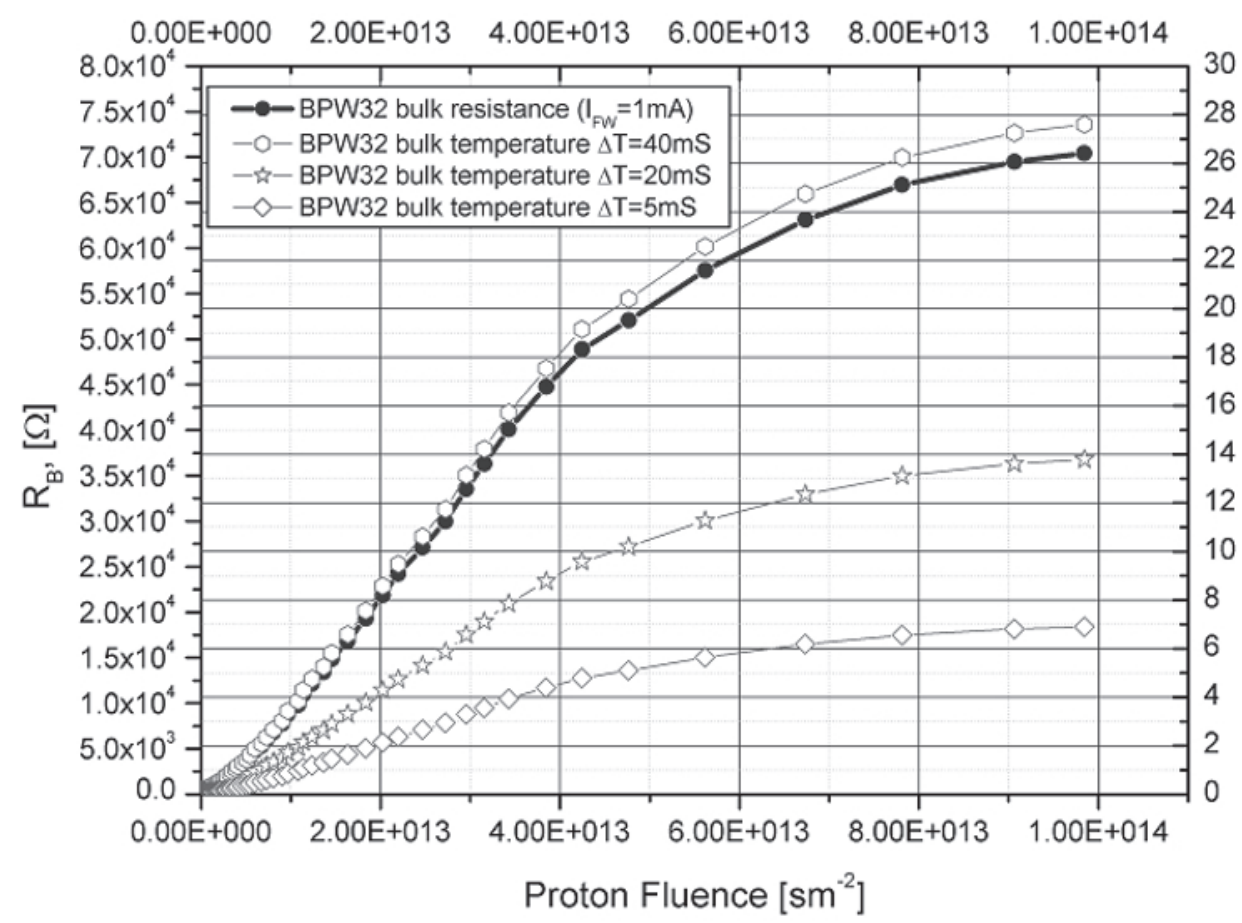

Figure 3: Temperature calculated for a BPW34 PIN diode according to [6] for different pulse lengths as function of proton fluence and the resistivity of the bulk as function of the proton fluence.

with known resistivity of the base. For the calibration of the PIN diode used in [6] it is necessary to use the same parameters of the test signal and the same ambient temperature.

The error of a measurement will increase with increasing absorbed dose and with increasing variation of the ambient temperature. The stabilization of the temperature of the PIN diode can be provided in two ways. The first one is decreasing the current across the diode during irradiation. The second one is decreasing the pulse length for a constant amplitude of the current. Using the experimental data from [6] we calculated the change in bulk resistivity for the BPW34 PIN diode during irradiation. Furthermore, we calculated the increase in temperature as function of the absorbed dose for different pulse times, and for constant amplitude of the current (Figure 3). The value of the temperature increase was obtained using equation (3) under the assumption of a stationary process without any transition effects. In case of a constant current pulse, we can calculate the length of the pulse from equation $\Delta T_{1}=\Delta T_{0} / K$. 
In order to obtain an optimal calibration the way to decrease the value of the current seems more preferable, because we can change the dynamic range of the voltage measurements without losing the precision of the dose measurement. From a practical point of view it is easy when the time length of pulse is variable. The algorithm of the calibration we will describe in the next chapter.

\section{Device hardware, software and firmware}

Our dosimeter was developed as a simple system with both a manual control in order to read the forward voltage drop across the PIN diode and a system control by an external personal computer (PC). In case of PC control the dosimeter allows for online monitoring of the diode that is under investigation and for displaying data as fluence of neutrons. The dosimeter can be used in many fields e.g. calibration of detectors and determination of their radiation hardness, and also for studying different materials exposed to high radiation doses.

\section{Hardware}

The main functions incorporated in the dosimeter are:

- a current generator (Curr. Gen.) allowing to set a stable current for the diode under investigation in range of $1-99 \mathrm{~mA}$ with steps of $1 \pm 0.1 \mathrm{~mA}$ via the digital switches DSW1, DSW2;

- a high voltage supply (HVS) that allows to generate $25 \mathrm{~V} \mathrm{DC}$ for the current generator;

- a 12 bit ADC to measure the voltage drop on the diode in the range $0-5 \mathrm{VDC}$ with an accuracy of $\pm 10 \mathrm{mV}$;

- a temperature sensor ( $T$ sens.) to measure the temperature in the range from -20 to $+80^{\circ} \mathrm{C}$ with an accuracy of $\pm 0.25^{\circ} \mathrm{C}$ and a resolution of $0.06^{\circ} \mathrm{C}$;

- a reference voltage of 1.25 VDC for the calibration of the ADC;

The connections of the modules are made of analog switches SW1-5. The control module is based on an MCU Atmega8 from ATMEL Co. that allows controlling all measurement processes, and to send the results of the measurement to an LCD display. The MCU allows also to communicate with the external PC. 


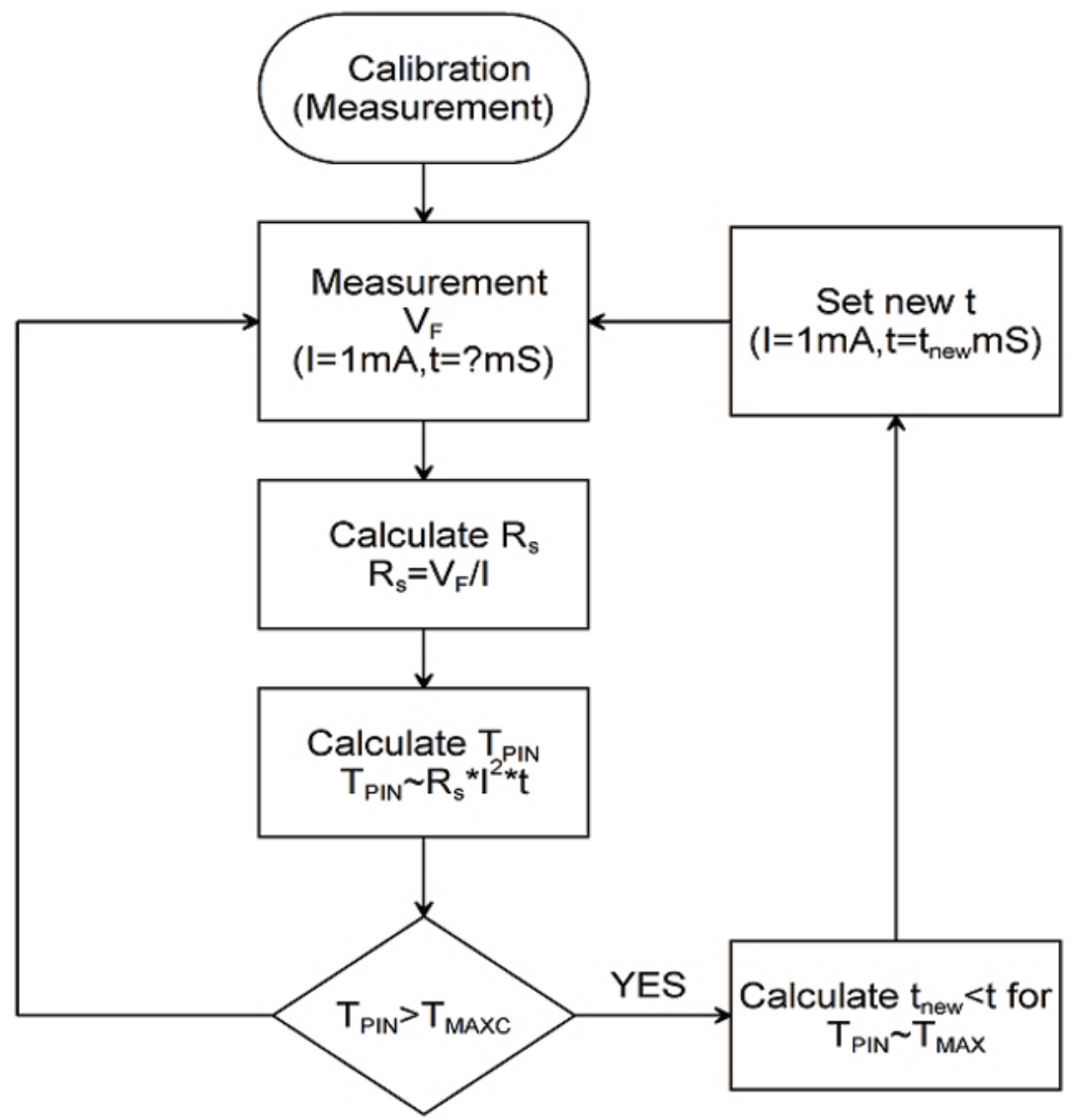

Figure 4: Flow chart of the optimal calibration/measurement algorithm.

\section{Firmware}

The firmware of the MCU Atmega8 was created using BASCOM AVR Basic. The algorithm for the optimal calibration and the measurements is shown in Figure 4. Different to standard methods of measurement our device has a variable time of the pulse. The pulse length is set to a value such that the temperature of the PIN diode bulk is not increasing more than $+2{ }^{\circ} \mathrm{C}$.

\section{Software}

The software for the control and the graphic user interface (GUI) of the dosimeter was created in the Lab Windows CVI environment. External 
control extends the usage of the dosimeter. First of all, using external software one can apply the dosimeter for online monitoring of the diode under investigation. Furthermore, one can use the embedded functions for sending alarm messages. One can select also the maximum limit of temperature, and the maximum value of measured voltage (fluence). When the maximum value is reached a short message can be sent to a pre-defined e-mail address. For calibrated diodes we can apply corrections for self-annealing, for the resistivity of long cables and for temperature fluctuations during the measurement.

For a known spectrum of radiation we can use a recalculation using the NIEL hypothesis.

\section{Conclusion}

Our dosimeter RM20 allows to read absorbed doses using a PIN diode. It is a practical device for application in a wide area of nuclear physics experiments, and for industrial and environmental monitoring. The current version of this device is using the optimized commercial PIN diode BPW34, and Si-1, Si-2 [7] as active elements. Automatic corrections have been used for the recalculation of dose in case of self-annealing of the BPW34. Automatic optimization and correction can be used for the adjustment of the pulse width of the BPW34 and the Si-2. The RM20 dosimeter can be controlled manually or by computer. This allows for remote monitoring of absorbed dose and for calibration of new types of diodes.

\section{Acknowledgements}

I would like to acknowledge Dr. M. Majerle for permanent interest to this work and for his support. This work was supported by the Ministry of Education of the Czech Republic (grant LC 07048).

\section{References}

[1] J. Mengali, E. Paskell, R. W. Beck, and C. S. Peet, Proceedings of the Second Conference on Nuclear Radiation Effects on Semiconductor Devices, Materials and Circuits, Sept. 1959.

[2] John M. Swartz and Marlin O. Thurston, Analysis of the Effect of Fast-Neutron Bombardment on the Current-Voltage Characteristic of 
a Conductivity-Modulated $p-i-n$ Diode, Journal of Applied Physics 37 (1966) 745-754.

[3] R. Korde, A. Ojha et al., The effect of neutron irradiation on silicon photodiodes, IEEE Transactions on Nuclear Science 36 (1989) 21692175 .

[4] A.B. Rosenfeld et al., PIN diode with a wide measurement range of fast neutron doses, Radiation Protection Dosimetry 33 (1990) 75-178.

[5] Dante M. Tasca, Pulse modes in failure power semiconductor, IEEE Transactions on Nuclear Science 17 (1970) 364-372.

[6] Ravotti T., et al., "BPW34 commercial p-i-n diodes for high-level $1 \mathrm{MeV}$ neutron equivalent fluence monitoring", in 9th European Conference on Radiation and Its Effects on Components and Systems, RADECS 2007, 10-14 September 200\%.

[7] http://www. cmi .cz/index $\cdot$ php?lang=1\&wdc=352. 